\title{
Improved Spin-Dependent WIMP Limits from a Bubble Chamber
}

\author{
E. Behnke ${ }^{1}$, J.I. Collar ${ }^{2 *}$, P.S. Cooper ${ }^{3}$, K. Crum ${ }^{2}$, M. Crisler ${ }^{3}$, M. $\mathrm{Hu}^{3}$, \\ I. Levine ${ }^{1}$, D. Nakazawa ${ }^{2}$, H. Nguyen ${ }^{3}$, B. Odom ${ }^{2}$, E. Ramberg ${ }^{3}$, \\ J. Rasmussen ${ }^{2}$, N. Riley ${ }^{2}$, A. Sonnenschein ${ }^{3}$, M. Szydagis ${ }^{2}$ and R. Tschirhart ${ }^{3}$ \\ ${ }^{1}$ Department of Physics and Astronomy, \\ Indiana University South Bend, South Bend, IN 46634, USA \\ ${ }^{2}$ Department of Physics, Enrico Fermi Institute and Kavli Institute for Cosmological Physics, \\ University of Chicago, Chicago, IL 60637, USA \\ ${ }^{3}$ Fermi National Accelerator Laboratory, \\ Batavia, IL 60510, USA
}

*To whom correspondence should be addressed; E-mail: collar@uchicago.edu

Bubble Chambers provided the dominant particle detection technology in accelerator experiments for several decades, eventually falling into disuse with the advent of other techniques. We report here on the first period of operation of an ultra-clean, room-temperature bubble chamber containing 1.5 kg of superheated $\mathrm{CF}_{3} \mathbf{I}$, a target maximally sensitive to spin-dependent and -independent Weakly Interacting Massive Particle (WIMP) couplings. An exposure in excess of $250 \mathrm{~kg}$-days is obtained, with a live-time fraction reaching $80 \%$. This illustrates the ability to employ bubble chambers in a new realm, the search for dark matter particles. Improved limits on the spin-dependent WIMP-proton scattering cross section are extracted from this first period. An 


\section{extreme intrinsic insensitivity to the backgrounds commonly limiting these ex-}

periments (a rejection factor for photon-induced electrons of $\sim 10^{-10}$ ) has been measured in operating conditions leading to the detection of low-energy nuclear recoils such as those expected from WIMPs.

\section{Introduction}

With approximately $85 \%$ of the total matter of the universe in a form which still eludes direct detection, the need for large-mass, background-insensitive detectors able to explore the very small couplings expected from Weakly Interacting Massive Particles (WIMPs) is urgent. While a member of this family of hypothetical particles, the Lightest Supersymmetric Partner (LSP, a.k.a. neutralino) stands out as one of the most likely candidates for the Dark Matter in galaxies (1, 2), its predicted interaction rate via low-energy elastic scattering off nuclei is $<<1$ event/kg-day for any target. In some unfavorable, yet entirely plausible models this can become a dismayingly small $<1$ event/ton-year. To further aggravate this situation, the couplings still allowed by present direct searches are already so weak that the next generation of massive $(\mathrm{O}(100) \mathrm{kg})$ WIMP detectors will necessarily suffer from a penury of statistics in any dark matter signal they may observe. For instance, unique signatures such as a $\sim 5 \%$ annual modulation in WIMP interaction rate expected from the orbital motion of the Earth (3) will soon require extreme exposures of $\mathrm{O}(100)$ Ton-year to become evident. Information about WIMP properties (e.g., mass of the particle) will be scarce. This clearly indicates the need for a variety of detection techniques and targets, and for an emphasis within each experiment not only on improving sensitivity, but on developing schemes that allow the unequivocal identification of these particles (4).

Superheated liquids, in the form of bubble chambers, were extensively used as the detection medium of choice in accelerator experiments spanning more than three decades. These 
chambers led not just to numerous discoveries, but also to an effect nearly as important: an unprecedented ability to visualize fundamental particles and their interactions (5, 6). The advent of newer technologies led to their progressive obsolescence during the seventies. In a bubble chamber, the heat deposited by ionizing radiations along their path produces local nucleations of the vapor phase in a delicate (metastable) superheated liquid. Rapidly growing bubbles form along this path, are photographed, and the chamber is then reset by fast recompression to the stable liquid phase. Decompression follows, bringing the target below its vapor pressure at the operating temperature, sensitizing it to radiation and starting the cycle anew. In accelerator experiments it was sufficient to maintain the superheated state during the few milliseconds corresponding to the bunched arrival of incident particles. Uncontrollable boiling on porous materials (gaskets, metallic surfaces) prevented serious consideration of this type of detector in searches for rare events, where the time of arrival of the signal is not known.

Taking several precautions towards the deactivation of inhomogeneous bubble nucleation centers, we have recently shown that it is possible to achieve what is in principle an indefinitely long stability in moderately superheated bubble chambers (7), thereby allowing their use in direct WIMP searches. In this realm of application, several advantages for this type of detector can be mentioned. Most important is the fact that the superheated liquid can be tuned to respond exclusively to particles having a large stopping power $(d E / d x$, energy loss per unit path distance). In this way muons, gamma rays, $\mathrm{x}$-rays, beta particles, etc., all fall well below a bubble nucleation threshold, which is typically $d E / d x>50 \mathrm{keV} / \mu \mathrm{m}$ during a WIMP search (Fig. 1). The target liquids are nevertheless sensitive to few-keV nuclear recoils such as those expected from the scattering of WIMPs, given their much denser energy deposition. The process of radiation-induced bubble nucleation is described within the framework of the classical "Hot Spike" model (8): for the phase transition to occur, the energy deposited by the particle must be larger than the thermodynamical work of formation of a critically-sized protobubble, 
but this energy must also be lost over a distance comparable to the size of this protobubble, i.e., a minimum stopping power condition must be fulfilled. This condition leads to an advantageous insensitivity to the listed minimum ionizing backgrounds that normally plague WIMP searches (Figs. 1,2). Formal details on the theory of radiation-induced bubble formation in superheated fluids can be found in $(9,10,11)$ and references therein. As for the probability of spontaneous (homogeneous) bubble nucleation in the bulk of a superheated fluid, it only becomes sizeable a few degrees below the critical temperature of the target compound (12). In normal operating conditions for a dark matter search this source of instability is entirely negligible.

\section{Experimental}

Chamber operation Early in 2005 we installed a chamber containing $1.5 \mathrm{~kg}$ of $\mathrm{CF}_{3} \mathrm{I}$, an industrial refrigerant commonly used as a fire extinguisher, at the 350-foot depth of the NuMI (Neutrinos at the Main Injector) tunnel in Fermilab. Environmental neutrons can produce single bubbles such as those expected from WIMPs if they scatter just once in the target (Fig. 2). At this shallow depth, a combination of active and passive shielding can lead to a neutron background rate as low as 0.01 bubbles / $\mathrm{kg}$-day. The choice of $\mathrm{CF}_{3} \mathrm{I}$ provides optimal sensitivity to both spin-dependent (SD) and spin-independent (SI) WIMP couplings, in the first case by its fluorine content, in the second via the presence of iodine $(1,2,13)$. This allows a maximally efficient exploration of supersymmetric WIMP candidates (4).

Bubble production within the chamber is monitored via two trigger mechanisms, the sound emission or pressure rise created by their formation and growth, and by the changes that their appearance induces in CCD camera images, inspected every $\sim 20 \mathrm{~ms}$. Two orthogonal cameras watch the inner volume (Fig. 2), where the active liquid resides within a thin quartz vessel. A bellows mechanism balances the pressure difference across the quartz vessel wall (14). The precision in the stereoscopic reconstruction of the spatial position of small early bubbles is of 
the order of their size at the time of the trigger, $\sim 1 \mathrm{~mm}$. A live-time fraction of $\sim 80 \%$ was obtained after the adoption of real-time image analysis as the primary trigger.

The chamber was operated continuously, in a variety of conditions (pressure, temperature, presence of radioactive sources, different triggering methods and pressure cycling protocols), for a year starting on December 2005, generating an exposure larger than $250 \mathrm{~kg}$-days, with an overall mean time between expansions of $\sim 100 \mathrm{~s}$. The emphasis on this first large prototype was purely on the functional aspects leading to continuous unattended operation and enhanced stability, obtained by implementing the techniques described in (7). In the interest of rapid deployment, little attention was paid to alpha-recoil backgrounds such as those induced by radon and its progeny (Fig. 1). The data at hand, although entirely dominated by radon-induced backgrounds, already yield an improved sensitivity to SD WIMP-proton couplings.

Calibrations Of importance prior to a WIMP search is an empirical determination of the maximum degree of superheat (15) achievable before the onset of sensitivity to minimum ionizing particles such as photoelectrons (Fig. 1). This in turn defines the lowest recoil energy that can be detected in background-free conditions, and with it the acceptance for a WIMP signal. Similarly, it was necessary to demonstrate that sensitivity to low-energy nuclear recoils was nevertheless present in those conditions. Two calibration sources were developed for these purposes. The first one, an intense $13 \mathrm{mCi}{ }^{137} \mathrm{Cs}$ gamma source, was placed next to the chamber's outer steel vessel, inside its neutron-moderating polyethylene shield. Fig. 3 shows the response of the chamber as a function of operating pressure in the presence of the source and in its absence. A Monte Carlo simulation (16) was used to calculate the rate of photon-induced electron production within the active volume of the chamber. From the difference between this interaction rate and the observed bubble nucleation rate in the presence of the source it is possible to obtain a gamma rejection factor (the fraction of interacting gammas inducing bubbles) as a 
function of degree of superheat. This factor can then be expressed in terms of the calculated nuclear recoil energy threshold for each pressure setting (Fig. 3, inset). The minimum-ionizing background rejection obtained, $\sim 10^{-10}$ for a nuclear recoil threshold of $\sim 10 \mathrm{keV}$, is unmatched by any other WIMP detector. For instance, dark matter detection efforts using cryogenic germanium detectors sensitive to both ionization and heat (Akerib et al. in $(\sqrt{13})$ ) presently feature a rejection factor $10^{-4}-10^{-5}$ for a similar recoil threshold. As a result of this, moderately superheated bubble chambers are free from any radiopurity constraints in gamma or beta emitters in the detector or its neutron moderator shield, including possible elevated rates of ${ }^{14} \mathrm{C}$ in the active liquids. This freedom, when combined with room-temperature operation, translates into large gains in construction speed and a reduction in costs. This background insensitivity is intrinsic: electron-induced events simply do not take place.

The second calibration source is a switchable Am/Be neutron source (Fig. 4). It was characterized using a ${ }^{3} \mathrm{He}$ counter surrounded by moderator. A Monte Carlo simulation (16) was used to generate the response of the counter, leading to a measured neutron yield of $4.9 \mathrm{n} / \mathrm{s}$, in excellent agreement with the $(\alpha, n)$ reaction yield from the ${ }^{241} \mathrm{Am}$ foil sources employed and separately characterized. In order to further assess the uncertainty in the very small yield of the source (19), the same simulation package and neutron detector were used to characterize five commercial neutron sources of known activity. This uncertainty was found to be a modest $\pm 11 \%$. This switchable source, also placed outside of the steel wall of the recompression chamber, is moderated by the $\sim 10 \mathrm{~cm}$ of recompression fluid around the quartz vessel and is therefore expected to produce recoils very similar in energy to those from WIMP interactions (Fig. 4, inset). In comparing the predicted response to this source with actual observations, one must take into account additional sources of uncertainty affecting the input to the MCNP-PoliMi Monte Carlo (21) used to transport neutrons and generate the expected rate and energy distribution of recoils in the active liquid. We have appraised these to be dominated by a $\pm 28 \%$ from the 
fiducialization of the chamber geometry and a $\pm 24 \%$ from the hardness of the neutron spectrum (affected by alpha-particle energy losses on their way to the Be foils (22)). The comparison is very good, as can be appreciated in Fig. 4. These predictions were generated prior to inspection of the neutron irradiation data. The success of this calibration confirms the predictions for bubble nucleation thresholds as a function of superheat, sanctioning WIMP limits obtained from the device. Switchable sources similar to this one can be used in planned larger chambers for periodic studies of response to nuclear recoils, important for instance when looking for WIMPinduced modulations in the data (3). To the best of our knowledge this is the first instance of their use in a direct search for WIMPs.

Present limitations of the method Two backgrounds became evident during underground operation of the chamber. The first is an excess of bubbles on the wall of the quartz vessel. A fraction of their rate may arise from the exposure of the vessel to typical concentrations of radon in air before installation. This results in the shallow implantation of its long-lived alpha-emitting daughters (20). Another source able to explain most to all of the observed surface-alpha rate is the $\sim 50 \mathrm{ppb}$ of uranium measured via gamma spectroscopy in the quartz vessel material. An origin in alpha emission for these wall events is confirmed by their disappearance at pressures higher than 65 (35) psig, the predicted thresholds for alpha-induced bubble nucleation when operating at 40 (30) C. Image analysis readily identifies these bubbles as happening on the wall, allowing rejection with high confidence. However, since each event is followed by tens of seconds of recompression time to ensure recovery of the superheated state in the subsequent decompression (7), an excessive wall rate per unit quartz surface would lead to a considerable dead time in larger chambers. A decrease by $\times 10(\times 100)$ in the wall event rate from the presently observed value is needed to ensure that $\mathrm{O}(50) \mathrm{kg}$ chambers under construction sustain a live time $>60 \%$ (>85\%). In order to achieve this reduction, an etching procedure has been developed 
in collaboration with the vessel manufacturer, facilitating the removal of implanted daughters while preserving surface smoothness and resistance against fracture initiation. In addition, new chambers employ synthetic fused silica containing only a few ppt of alpha-emitters.

The second source of background events from this mechanical prototype detector consists of temperature-dependent radon emanations and injections into the chamber, likely from thoriated weld lines and a Viton O-ring exposed to the inner detector volume. Viton is known to release radon at a rate of $\sim 300 \mathrm{mBq} / \mathrm{m}^{2}$ and does not create a strong barrier against its diffusion from external sources. Radon decays can lead to single bubbles in the bulk identical to those expected from WIMPs (they nevertheless display a very different spectral and temporal dependence, as discussed below). That the origin of essentially all presently-observed bulk events is in radon-associated alphas and alpha-recoils is evidenced by two signatures. First, the distribution of times between consecutive bulk events (Fig. 5) displays a clear short-lived component corresponding to the the decay sequence ${ }^{222} \mathrm{Rn} \rightarrow{ }^{218} \mathrm{Po} \rightarrow{ }^{214} \mathrm{~Pb}$ (both steps are alpha-emitting). Two independent analyses of this distribution, including the full decay chain (Fig. 5), the effect of recompression (dead) time, fiducial volume cuts and the possibility of other sources being present, strongly favor models with $\sim 100 \%$ of the events belonging to the ${ }^{222} \mathrm{Rn}$ sequence and a $\sim 100 \%$ bubble nucleation efficiency for the combined alpha and alpha-recoil emission. Second, the distribution of bulk event rates vs. operating pressure at a fixed temperature is flat below a sharply defined onset pressure, the value of which is again in good agreement with theoretical expectations (Fig. 5). This is the kind of spectral behavior expected from the response of a threshold detector to a mono-energetic source. It has been observed in the past in bubble chambers intentionally spiked with alpha emitters (23). New larger chambers under construction use metallic seals, with all weld lines being non-thoriated, among other precautions against $\mathrm{Rn}$. 


\section{Improved sensitivity to WIMP couplings}

Even constrained by the background limitations of the mechanical prototype in these early runs, an improved sensitivity to the spin-dependent WIMP-proton coupling is obtained from these data. This is possible due to the extreme sensitivity to this mode of interaction afforded by the large mass fraction of fluorine $(29.1 \%)$ and the operation of the chamber in conditions where only nuclear recoils (alpha, neutron or WIMP-induced) can produce bubbles. Fig. 5 displays the characteristic recoil rate expected from example WIMPs, overlaid on the data. Such spectra are calculated for different WIMP masses using the theoretical bubble nucleation thresholds (top axis in the figures) for fluorine recoils (24). These are in turn used to generate the integrated WIMP recoil rate above threshold, following the method and dark matter halo parameters recommended in (25). These threshold calculations have been validated by their success in predicting the response of the chamber to the Am/Be source (26). Following a procedure similar to that in (27), experimental bulk bubble nucleation rates vs. pressure, such as those depicted in Fig. 5, are fitted with a model containing two free parameters: the signal from a WIMP of a given mass, scalable by a free spin-dependent cross-section, and the response to ${ }^{222} \mathrm{Rn}$ alphas, expressed as a logistic function with a free overall normalization (Fig. 5). The minimization is performed using the MINUIT package (28), its output cross-checked via a standard error matrix analysis. The best fits favor the null hypothesis alone (response to ${ }^{222} \mathrm{Rn}$ alphas only). The largest spin-dependent couplings allowed by the data, up to a $90 \%$ C.L., are displayed in the form of exclusion plots in Fig. 6. These bounds arise from the weighted average (25) of the analyses performed on three independent large data sets adding up to $52 \mathrm{~kg}$-days of exposure. 


\section{Conclusions}

Even prior to any measures against alpha-emitting backgrounds, improved bounds on spindependent WIMP couplings can be obtained from the application of an old technology, bubble chambers, to a new problem, that of dark matter detection. In particular, these new bounds make a spin-dependent interpretation (30) of the DAMA claim of an observed WIMP signal (31) very difficult. Other experiments have already severely constrained spin-independent explanations.

In principle, and strictly from the point of view of internal backgrounds, the sensitivity of this technique can be improved by as much as six orders of magnitude by reducing alphaemitting contaminants from the uranium and thorium chains to levels similar to the best achieved in large neutrino experiments $\left(<10^{-17} \mathrm{~g} / \mathrm{g}\right)(34)$. This would guarantee an extensive probing of supersymmetric WIMP models along both spin-dependent and -independent couplings (4). The possibility exists to add the detection of dark matter to the many contributions already made by Bubble Chambers to particle physics. We presently concentrate on the development of radon-free chambers with a total target mass of $\mathrm{O}(50) \mathrm{kg}$.

We gratefully acknowledge the effort and outstanding technical support of the Fermilab Staff. This work is supported by NSF CAREER award PHY-0239812, NSF grant PHY-0555472, the Indiana University South Bend R\&D committee, the Kavli Institute for Cosmological Physics through grant NSF PHY-0114422, and the U.S. Department of Energy.

\section{References}

1. G. Jungman et al., Phys. Rept. 267, 195 (1996).

2. R.J. Gaitskell, An. Rev. Nucl. Part. Sci. 54, 315 (2004).

3. A.K. Drukier et al., Phys. Rev. D33, 3495 (1986). 
4. G. Bertone et al., Phys. Rev. Lett., in press, (arXiv:0705.2502).

5. D.A. Glaser, Nucl. Phys. B (Proc. Suppl.) 36, 3 (1994).

6. C. Rubbia, Nucl. Phys. B (Proc. Suppl.) 36, xvii (1994).

7. W.J. Bolte et al., Nucl. Instr. Meth. A577, 569 (2007).

8. F. Seitz, Phys. Fluids 1, 1 (1958).

9. J.I. Collar, Phys. Rev. D54, R1247 (1996).

10. J.I. Collar et al., Phys. Rev. Lett. 85, 3083 (2000).

11. J.I. Collar et al., New J. Phys. 2, 14.1 (2000).

12. M. Blander et al., A.I.Ch.E. Journal 21, 833 (1975).

13. The nuclear structure of Fluorine leads to the best expectations for SD WIMP couplings to protons (J. Ellis et al., Phys. Lett. B263, 259 (1991)). Large nuclei such as Iodine profit from a coherent enhancement to their SI cross section (1, 2). The importance of the SD channel has been recently emphasized (D.S. Akerib et al., Phys. Rev. D73, 011102(R) (2006), and refs. therein).

14. The illumination, imaging and pressure compensation systems of this chamber resemble those in the first hydrogen bubble chamber (D.E. Nagle et al., Rev. Sci. Instr. 27, 203 (1956)).

15. "Degree of superheat" is generally defined as the difference between the vapor pressure at the operating temperature and the operating pressure, i.e., is a measure of how far from equilibrium the compound is, and therefore of instability or sensitivity to radiation. $\mathrm{CF}_{3} \mathrm{I}$ is only moderately superheated at room temperature, with a vapor pressure $\sim 50$ psig, 
leading to the need to run the chamber at slightly higher temperatures (30-40 C) to achieve thresholds in recoil energy as low as few keV.

16. MCNP, A General Monte Carlo N-Particle Transport Code, J.F. Briesmeister ed., Los Alamos National Laboratory, LA-12625-M, 1993.

17. M. Das et al., Nucl. Instr. Meth. A531, 577 (2004), and references therein.

18. C.R. Bell et al., Nucl. Sci. Eng. 53, 458 (1974).

19. In shielded underground conditions this weak neutron source is sufficient to increase the present bulk bubble nucleation rate by a factor of four.

20. R. S. Lively et al., Health Phys. 64, 485 (1993).

21. S.A. Pozzi et al., Nucl. Instr. Meth. A513, 550 (2003).

22. K.W. Geiger et al., Nucl. Instr. Meth. 131, 315 (1975).

23. F.H. Poesposoetjipto et al., Helv. Phys. Acta 43, 203 (1970).

24. The small ( $20 \%$ or less) contribution from iodine to this coupling is also included. Spin parameters for Iodine (Bonn A model) are taken from M.T. Ressell et al., Phys. Rev. C56, 535 (1997).

25. J.D. Lewin et al., Astropart. Phys. 6, 87 (1996). A local WIMP density of $0.3 \mathrm{GeV} / \mathrm{cm}^{3}$, halo velocity dispersion of $230 \mathrm{~km} / \mathrm{s}$, Earth-halo velocity of $244 \mathrm{~km} / \mathrm{s}$ and escape velocity of $650 \mathrm{~km} / \mathrm{s}$ are employed.

26. The classical theory of radiation-induced boiling (8, 18) predicts, perhaps naively, sharp (step function) bubble nucleation thresholds. In contrast to (27), where soft (sigmoidal) thresholds are invoked to interpret neutron calibrations, we have observed no need to 
deviate from the predictions of this theory. In dedicated experiments using smaller devices and neutron-induced recoils we have ascertained that, at least for a $\mathrm{CF}_{3} \mathrm{I}$ bubble chamber, thresholds are very close to step-like. The rapid onset of full response to alpha-recoils observed in this larger chamber (Fig. 5) is another indication of the same. Adoption of a sigmoidal threshold would result in an enhanced WIMP sensitivity, most noticeably for low WIMP masses.

27. M. Barnabe-Heider et al., Phys. Lett. B624, 186 (2005).

28. F. James, Procs. of the 1972 CERN Computing and Data Processing School, Pertisau, Austria, 10-24 September, 1972.

29. http://dmtools.brown.edu

30. C. Savage et al., Phys. Rev. D70, 123513 (2004).

31. R. Bernabei et al., Riv. Nuovo Cimento 26N1, 1 (2003).

32. D. Tovey et al., Phys. Lett. B488, 17 (2000).

33. F. Giuliani, Phys. Rev. Lett. 93, 161301-1 (2004).

34. Y. Kishimoto, et al., in "Topical Workshop on Low Radioactivity Techniques" LRT 2004, AIP Conf. Procs. 785, 193 (2005). 


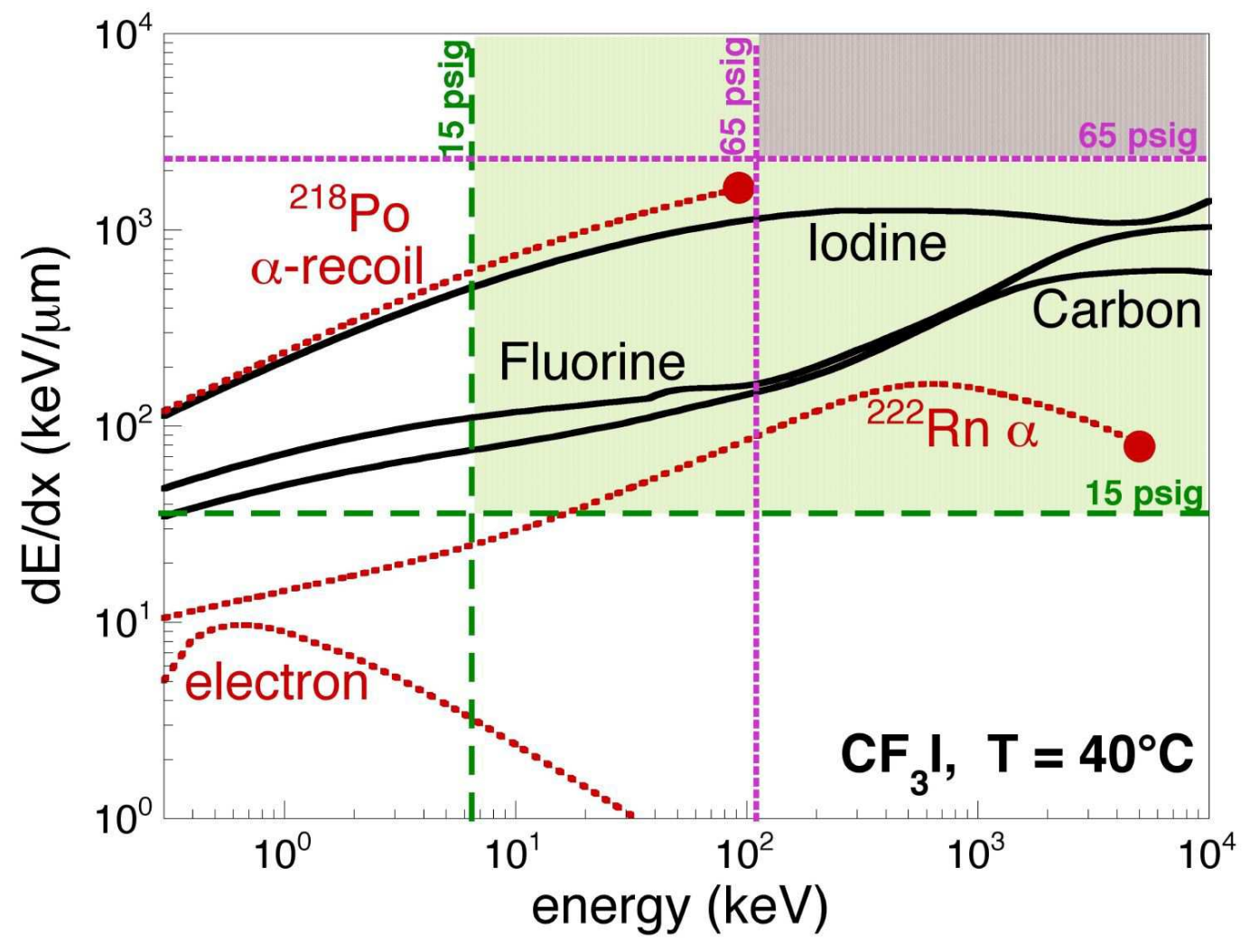

Figure 1: Instantaneous stopping power vs. energy for different particles in $\mathrm{CF}_{3} \mathrm{I}$, including its three recoiling species. Plotted as horizontal and vertical lines are the calculated $\mathrm{dE} / \mathrm{dx}$ and energy thresholds for bubble nucleation at $\mathrm{T}=40 \mathrm{C}$ and two different operating pressures. According to the "Hot Spike" nucleation model (8) only radiations in the top right (colored) quadrants can lead to bubbles. Notice the absence of this possibility for electrons even toward the end of their range, in conditions that nonetheless lead to a sensitivity to recoils of just a few keV, such as those expected from WIMP interactions. Alpha particles and their recoiling daughters can induce bubble nucleations and the presence of their emitters must therefore be avoided. 


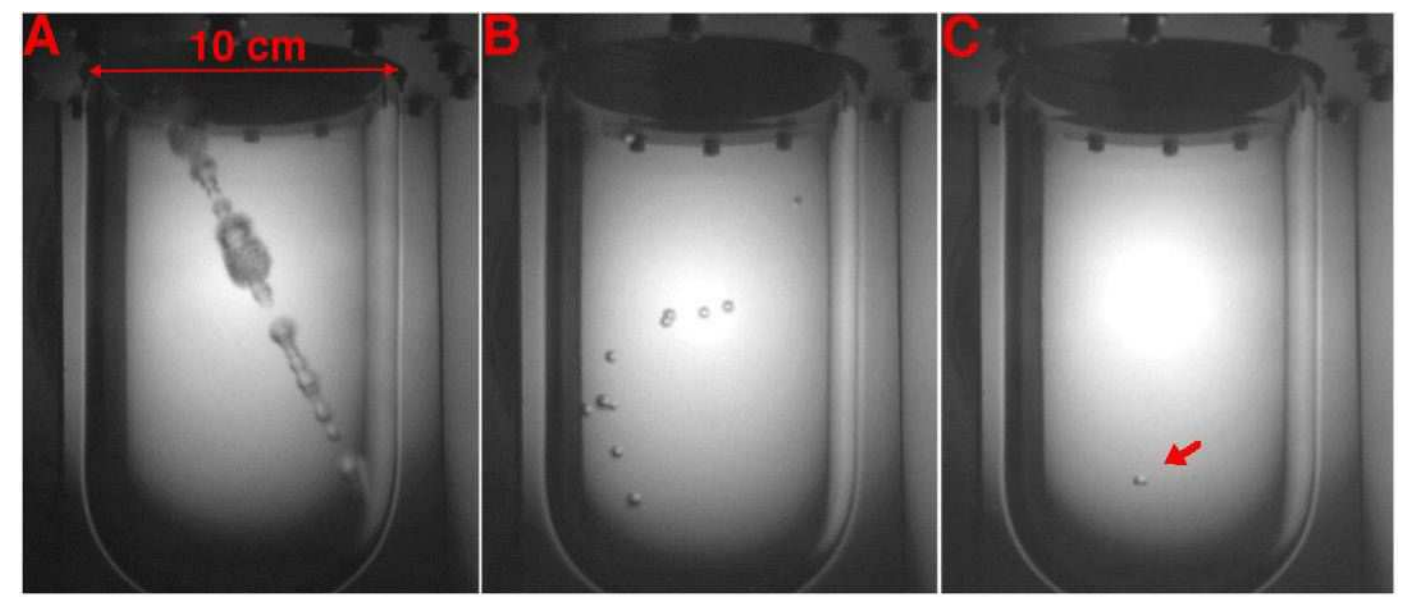

Figure 2: Families of events in a $1.5 \mathrm{~kg} \mathrm{CF}_{3} \mathrm{I}$ bubble chamber. At high degrees of superheat ( $\sim 60$ $\mathrm{C}$ and atmospheric pressure for this compound), minimum ionizing cosmic ray events reminiscent of those observed in early bubble chambers (5) are visible (left). At moderate superheats $(\sim 30 \mathrm{C}, 1 \mathrm{~atm})$ the chamber is sensitive strictly to high $d E / d x$ radiation such as nuclear recoils. Whereas neutrons can give rise to simultaneous separate bubbles each corresponding to a scatter (center), WIMPs are expected to produce single bubbles only (right), due to their extremely small probability of interaction. The mean free path between scatters is of just a few cm for neutrons, leading to an excellent ability to reject them in large chambers. 


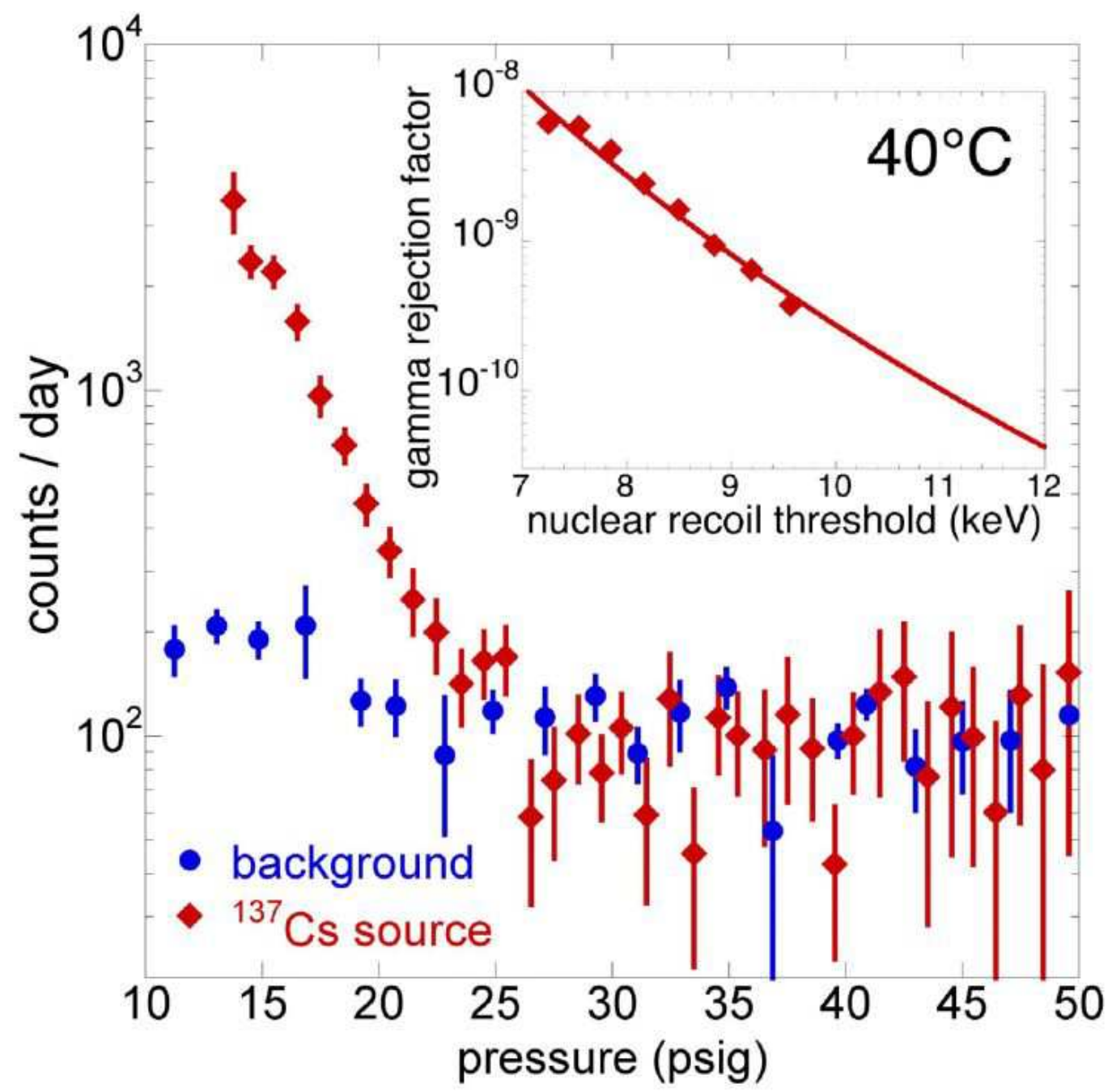

Figure 3: Response of the chamber to an intense ${ }^{137} \mathrm{Cs}$ gamma source. The expected gamma interaction rate with the superheated liquid is $3.9 \times 10^{6}$ per second, with gamma-induced electron energies reaching up to $662 \mathrm{keV}$. Inset: Intrinsic gamma rejection factor (fraction of interacting gammas inducing bubbles) obtained from the exposure to the source (see text). 


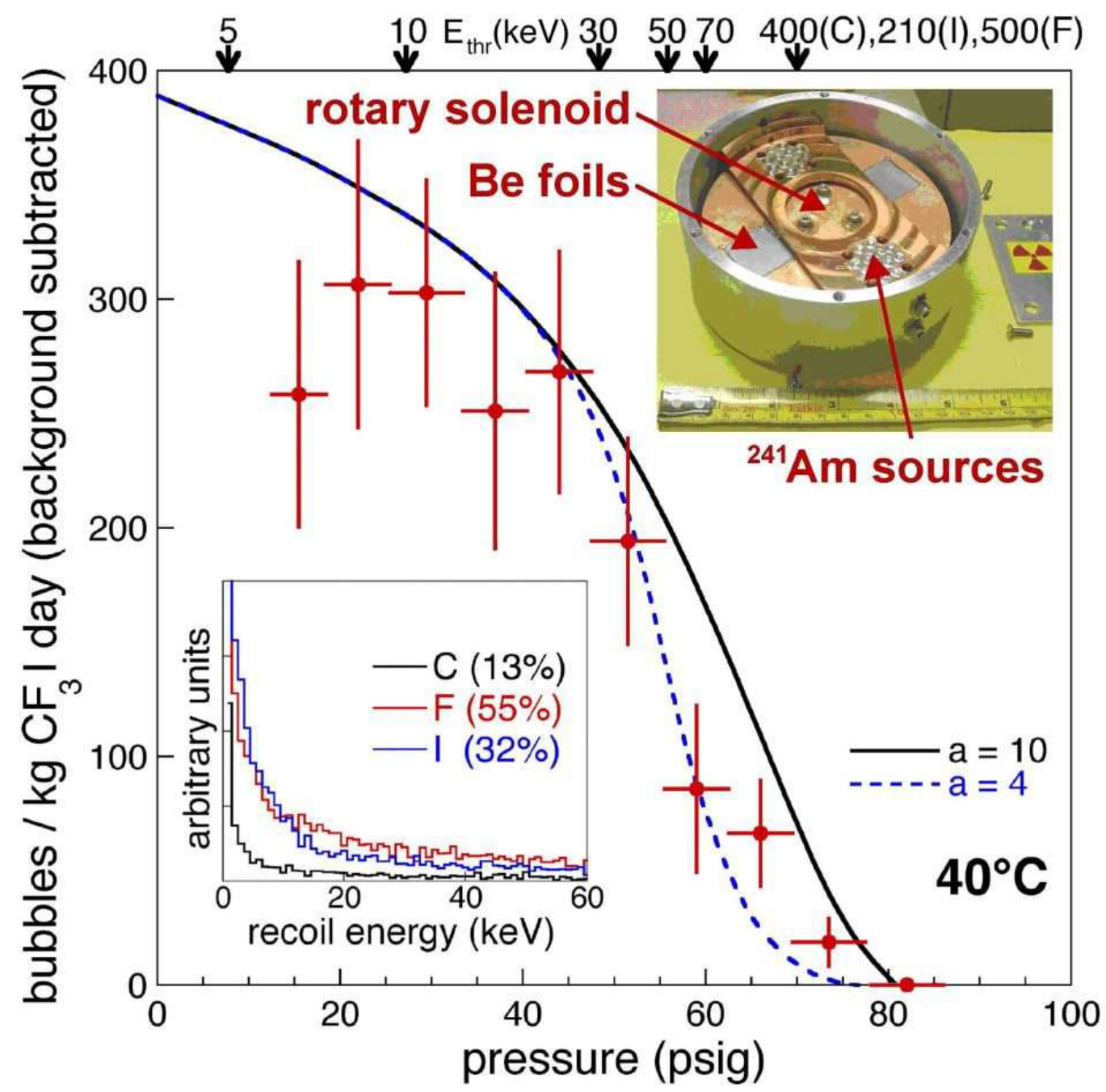

Figure 4: Blind absolute comparison between expected bubble nucleation rate (lines) and observations (points) in the active presence of the switchable Am/Be neutron source (top inset). The two lines correspond to largely different values of a nucleation parameter "a" (17), the single free factor in the classical theory used to predict bubble nucleation thresholds $(8)$. A fit to the data provides an excellent agreement with theoretical expectations $(a=6)(18)$. Including the uncertainty in the predictions (see text) the same fit simultaneously yields an efficiency in the response to this source of $81 \pm_{33}^{63} \%\left(51 \pm_{18}^{40} \%\right)$ at $40 \mathrm{C}(30 \mathrm{C})$ (errors are $90 \%$ confidence levels). Calculated thresholds for recoil-induced nucleation are expressed in $\mathrm{keV}$ along the top axis. Bottom inset: Spectrum of nuclear recoil energies produced by the source (MCNP-PoliMi simulation (21)), similar to typical expected WIMP recoil spectra. 

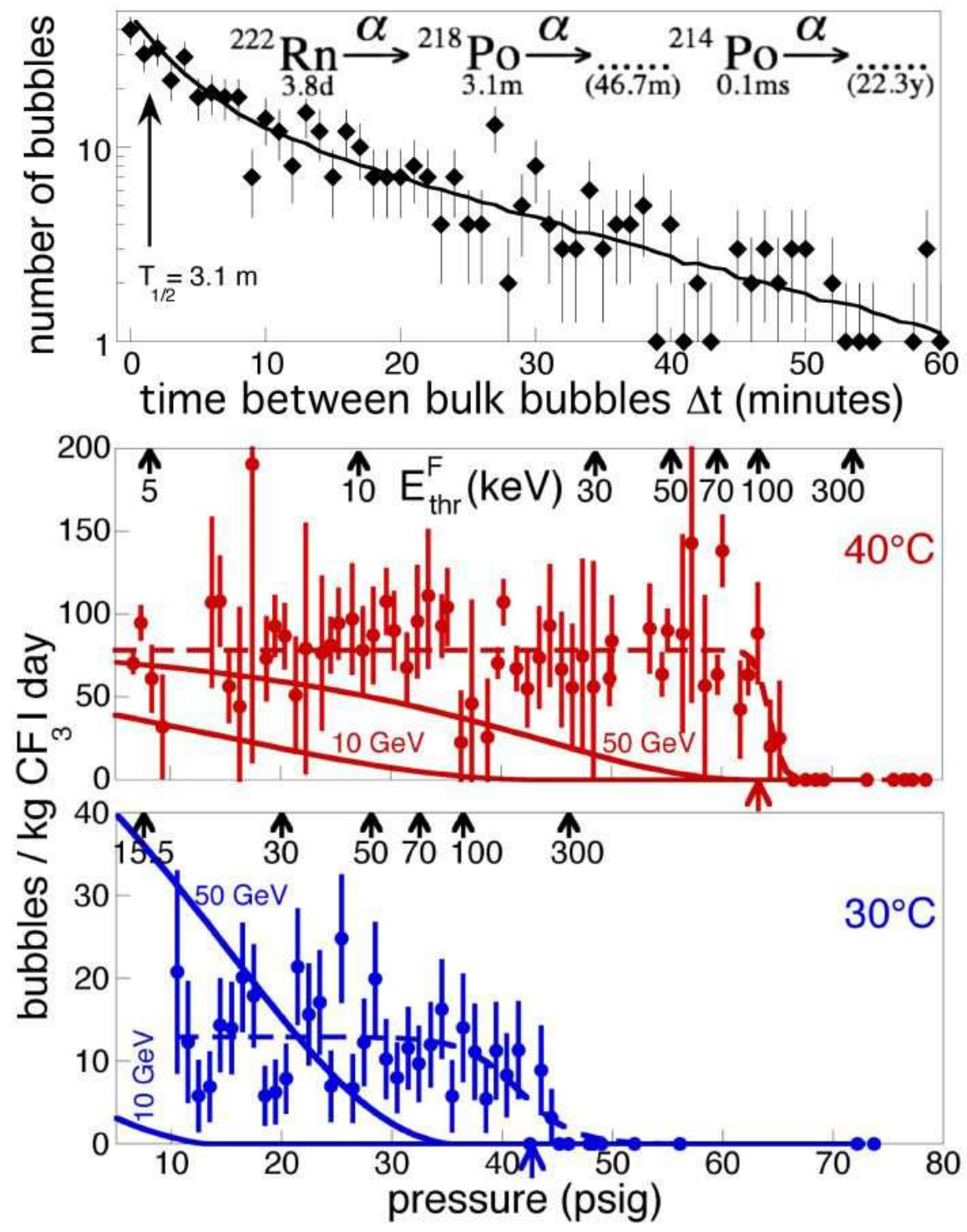

Figure 5: Top: Distribution of times between consecutive bulk events in the chamber. A model (solid line) including the effect of dead time and fiducial volume cuts, based on the triple alpha emission from ${ }^{222} \mathrm{Rn}$ and progeny, successfully reproduces this distribution (see text). Bottom: Distribution of single bulk bubble nucleation rate vs. operating pressure, for two different running temperatures. Rates display a flat behavior up to a pressure endpoint. This is characteristic of the response to monochromatic alphas and $\sim 100 \mathrm{keV}$ alpha recoils (here from ${ }^{222} \mathrm{Rn}$ emanations, see text). Colored arrows along the bottom axes indicate the predicted onset of sensitivity to these particles, in good agreement with observations. As a reference, solid lines correspond to the expected signal rate from WIMPs with a mass of 10 and $50 \mathrm{GeV} / \mathrm{c}^{2}$ and a $3 \mathrm{pb}$ cross section for their spin-dependent coupling to protons. Also shown is the response function to ${ }^{222} \mathrm{Rn}$ and progeny (dashed lines). Calculated energy thresholds (in $\mathrm{keV}$ ) for bubble nucleation by fluorine recoils are shown along the top axes. 

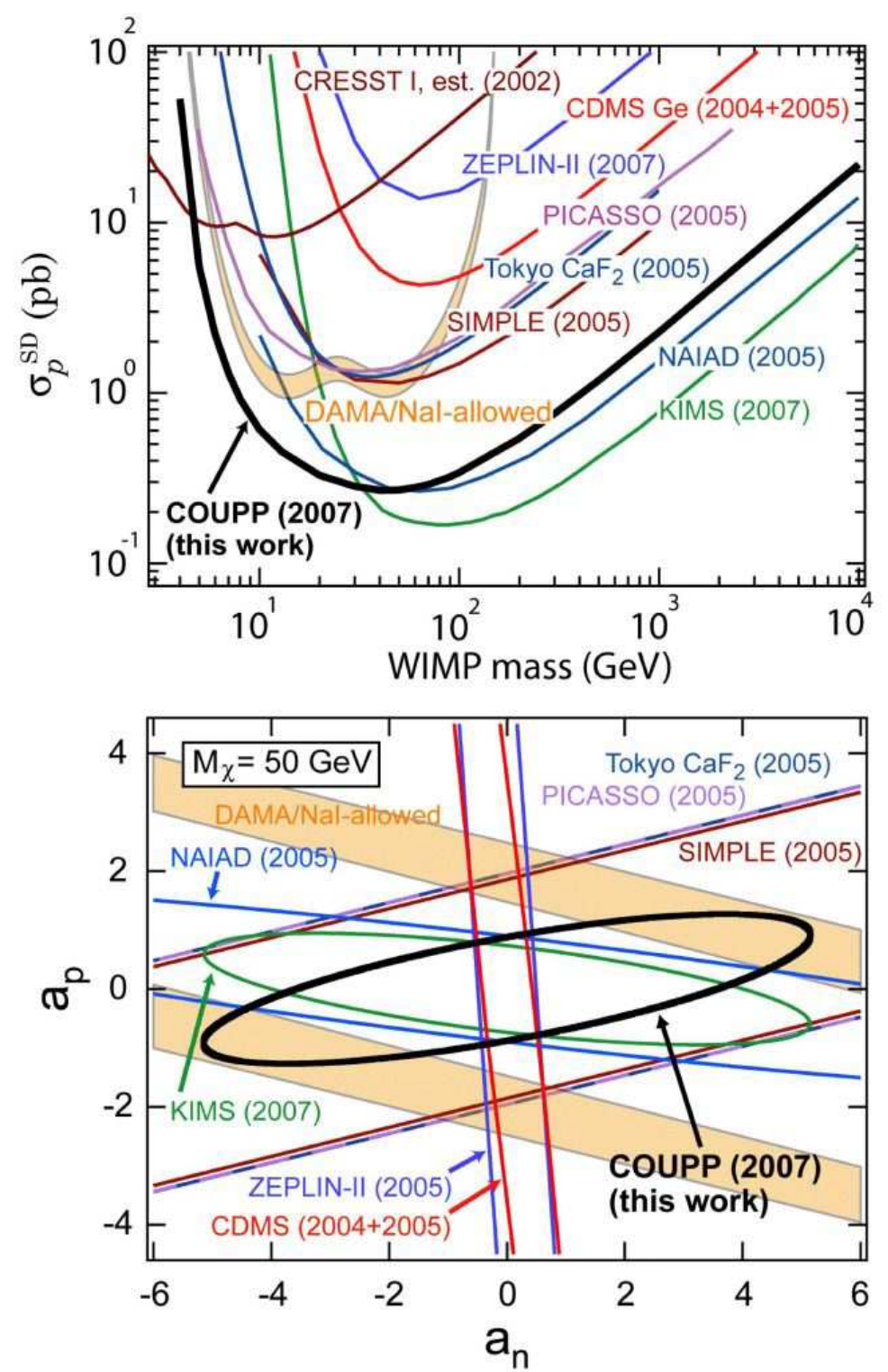

Figure 6: Top: Improved limits on spin-dependent (pure) proton-WIMP coupling vs. WIMP mass from this experiment (COUPP, the Chicagoland Observatory for Underground Particle Physics). Couplings above the line would have produced signals above observed backgrounds and are excluded to $90 \%$ C.L. Limits from other experiments are also shown (29), as well as the (orange) region favored as a possible explanation to an existing claim for WIMP observation (30. 31), a hypothesis now contradicted by this experiment. Bottom: Similar limits for spindependent coupling parameters where no assumption is made about the relative strength of the coupling to neutrons and protons, but a WIMP mass must be chosen $\left(50 \mathrm{GeV} / \mathrm{c}^{2}\right.$ here) (32, 33). The region outside of the ellipses is excluded by each experiment. 